$\underset{1}{9}$ DIAGNOSTIC PATHOLOGY

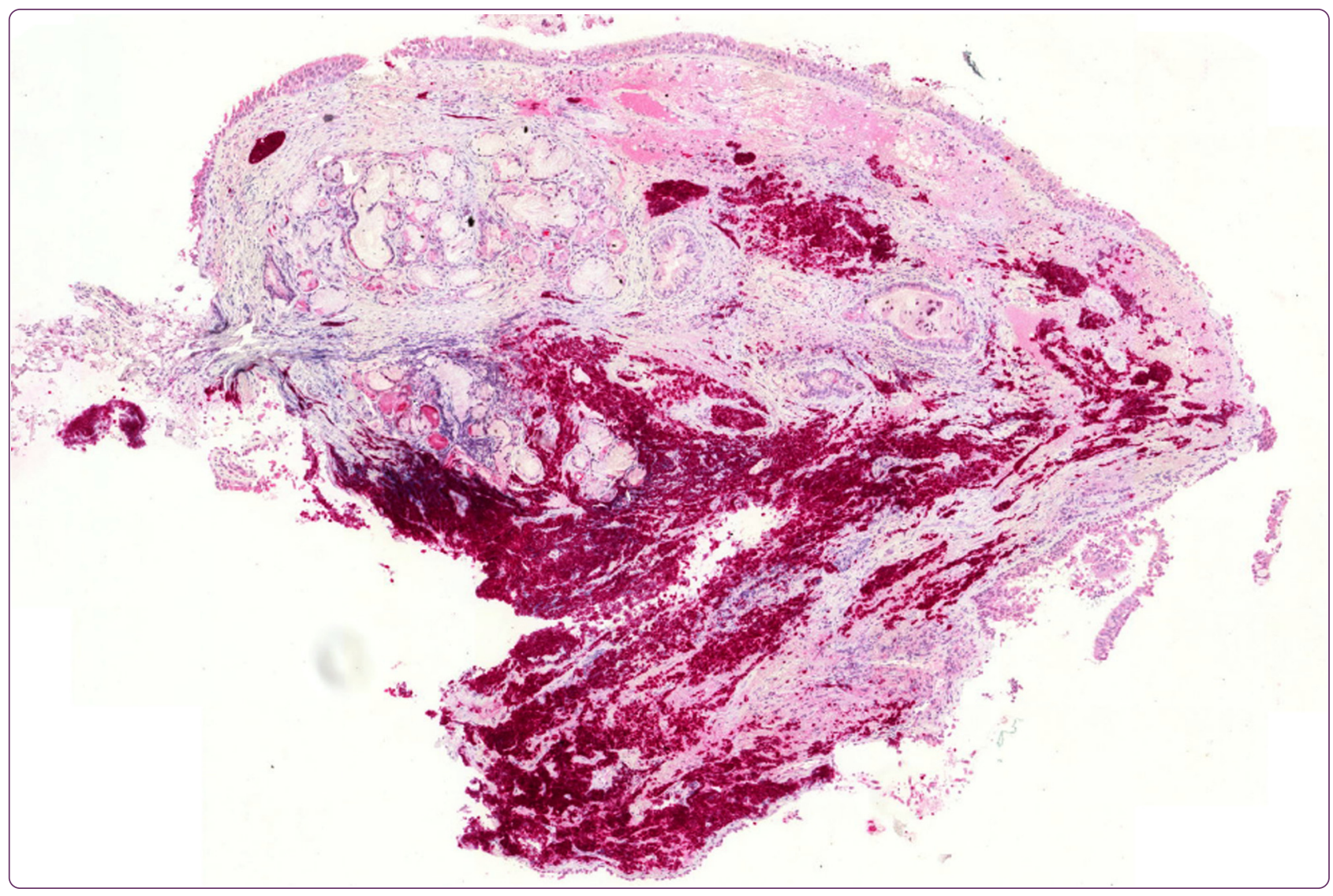

\title{
Value of thyroid transcription factor (TTF)-1 for diagnosis and prognosis of patients with locally advanced or metastatic small cell lung cancer
} Misch et al. 


\title{
Value of thyroid transcription factor (TTF)-1 for diagnosis and prognosis of patients with locally advanced or metastatic small cell lung cancer
}

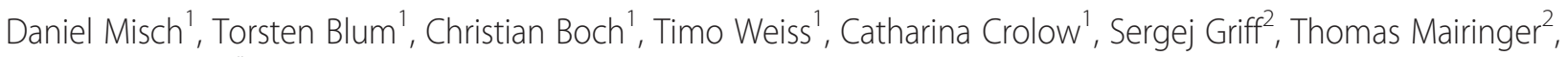
Torsten T Bauer $^{1 *}$ and Jens Kollmeier ${ }^{1}$

\begin{abstract}
Background: The aim of this study was to analyze the frequency of Thyroid Transcription Factor (TTF)-1 expression in small cell lung cancer (SCLC) and its value for the diagnosis of SCLC, the response to first line treatment as well as the prognostic impact on overall survival (OS).

Methods: We analyzed a total of 294 patients $(m, n=184 ; f, n=110)$ with SCLC (stage IIIA, $n=32 ;$ IIIB, $n=87 ; I V, n=175$ ) diagnosed in our institution between January 2005 and December 2008. Patient's characteristics comprising age, gender, histology and first line treatment were included into the analyses. For the follow-up of patients the governmental death registrar was used. The TTF-1 immunostaining was prospectively performed. $C T$ scans of all patients were reviewed and response to treatment was evaluated using the Response Evaluation Criteria In Solid Tumors 1.0 (RECIST) criteria.

Results: A total of 221 of the 294 patients were eligible for analysis. Patients with TTF-1-positive SCLC had a median OS of 374 (95\% Cl 306-442) days. The OS of patients with TTF1-negative SCLC was 290 (95\% Cl 191-389) days, which was not significantly shorter $(p=0.254)$. Also stratification for tumor stage did not reveal significant difference in OS. Analyzing the disease control rate (DCR) in patients with metastatic disease (stage IV), we observed a significantly $(p=0.006)$ improved response to treatment in the group of patients with TTF-1-expression (DCR 86\% vs. 56\%). Regarding the overall response rates (ORR) in the entire population, there was no difference observed between both subgroups. (TTF-1-pos. 75.3\% vs. TTF-1-neg. 71.4\%; $p=0.642$ ).
\end{abstract}

Conclusions: The diagnostic information of TTF-1 in SCLC seems to be limited. TTF-1 had no prognostic value concerning OS, but may serve as a predictor for response to first line chemotherapy.

Virtual slides: The virtual slide(s) for this article can be found here: http://www.diagnosticpathology.diagnomx.eu/vs/ 5811254651472285

Keywords: Small cell lung cancer, Histology, Thyroid transcription factor, Prognosis

\section{Background}

The DNA-binding protein thyroid transcription factor 1 (TTF-1) is mainly expressed in the thyroid gland and the lungs and plays an important role in the development of these organs. It is expressed in type II pneumocytes and Clara cells and has a crucial part in regulating the expression of various genes, such as for the surfactant or the Clara cell protein [1-5].

\footnotetext{
* Correspondence: torsten.bauer@helios-kliniken.de

'Department of Pneumology, Lungenklinik Heckeshorn, HELIOS Klinik Emil von Behring, Walterhöferstr. 11, 14165 Berlin, Germany

Full list of author information is available at the end of the article
}

It is known that the expression of TTF-1 is helpful to distinguish primary lung cancer from other nonpulmonary malignancies. The expression differs among the different histologic subtypes of lung carcinoma [6-9]. While TTF-1-expression was found to be frequent in adenocarcinoma and small cell lung cancer, it appears to be rare in patients with squamous or large cell carcinoma. In SCLC, the expression of TTF-1 was found in about $85-90 \%$ of cases [10]. Furthermore, it has been described that non-pulmonary small cell carcinoma can express TTF-1 due to their neuroendocrine differentiation [11]. Thus, the diagnostic value of TTF-1 in SCLC 
remains questionable. If it plays a role in the carcinogenesis of lung or thyroid cancer remains unclear, although there is evidence that it may be associated with the neuroendocrine differentiation of tumor cells [12].

Recent studies were addressed to the prognostic value of TTF-1-expression in lung carcinoma. Concerning NSCLC, TTF-1-expression is associated with better overall survival, which is even more pronounced in the subgroup of patients with adenocarcinoma [9,13-16]. A further aspect of any biomarker is its possible predictive value concerning response to chemotherapy. To our knowledge, there is currently no study which analyzed overall survival as well as response to first line chemotherapy of SCLC according to TTF-1-expression.

Therefore, the present study analyzes the frequency of TTF-1-expression in patients with locally advanced or metastatic small cell lung cancer and its prognostic value concerning overall survival as well as the predictive value for response to treatment.

\section{Methods}

\section{Patient's data and data acquisition}

We retrospectively analyzed 294 patients (male, $\mathrm{n}=184$; female, $n=110$ ) who were treated for histologically proven locally advanced or metastatic small cell lung cancer in our institution between January 2005 and December 2008. Patients characteristics were entered into a database and the following parameters were extracted for analysis: Age, gender, date of diagnosis, TNM classification (UICC 6) [17], initial therapy including chemotherapy and radiotherapy, response to first line treatment according to RECIST 1.0 [18] and the TTF-1 status of the diagnostic specimen. The TTF-1-immunostaining was performed prospectively using standard immunohistochemistry (Antibody Clone TTF-1 SP141, Ventana Medical Systems, DAB detection Kit). The immunostained cells were considered positive only when distinct nuclear staining was identified [19]. The tumor was identified as TTF-1 positive when more than $5 \%$ of cells stained for TTF-1. Further immunohistochemical markers stained were CD56, KL-1 or CKMNF-116 and MIB-1 [Figures 1 and 2].

Survival of these patients was followed up on a regular basis and mortality data were verified every six month with the governmental death registrar.

Data were gathered for internal quality control on a routine basis and all patients gave informed consent for data collection. The institutional review board (IRB-LungClinic Heckeshorn) therefore waived the need for ethical committee approval.

\section{Therapy}

Standard therapy in our institution comprised chemotherapy, radiation and their combination. Standard chemotherapy in all stages comprised cisplatin $(80-100 \mathrm{mg} / \mathrm{m} 2$ body surface, d1) or carboplatin (AUC 5 in cases with renal insufficiency with a glomerolar filtration rate $<60 \mathrm{ml} / \mathrm{h}$ ) in combination with etoposide $\left(100-140 \mathrm{mg} / \mathrm{m}^{2}\right.$ body surface, d1-3). A combination radiochemotherapy was considered in stage III and applied simultaneously or sequentially according to the performance status of the patients.
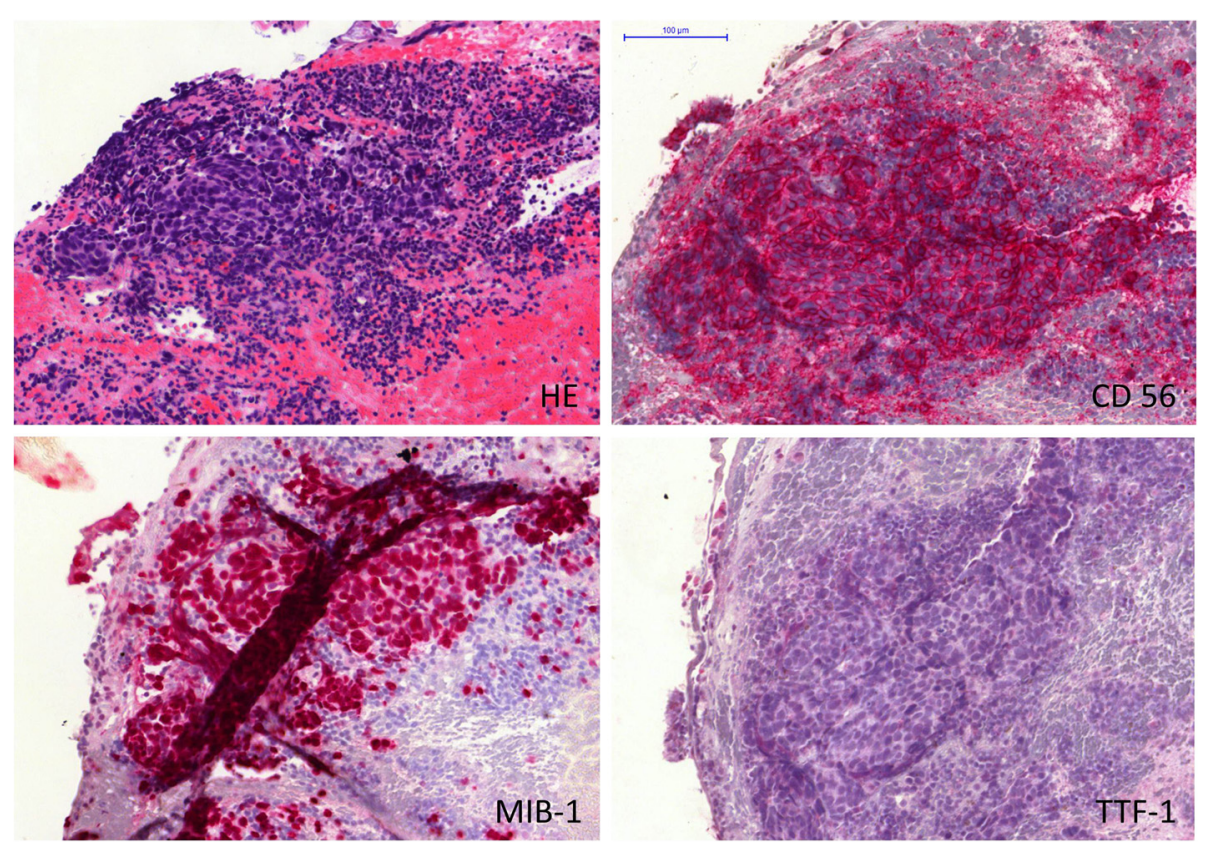

Figure 1 Example of TTF-1 negative small cell lung cancer (SCLC) staining. Left upper panel: Hematoxylin and eosin stain (HE). Right upper panel: Neural cell adhesion molecule (CD56). Left lower panel: Ki-67 Proliferation marker (Clone MIB-1). Right lower panel: Thyroid transcription factor-1 (TTF-1). 

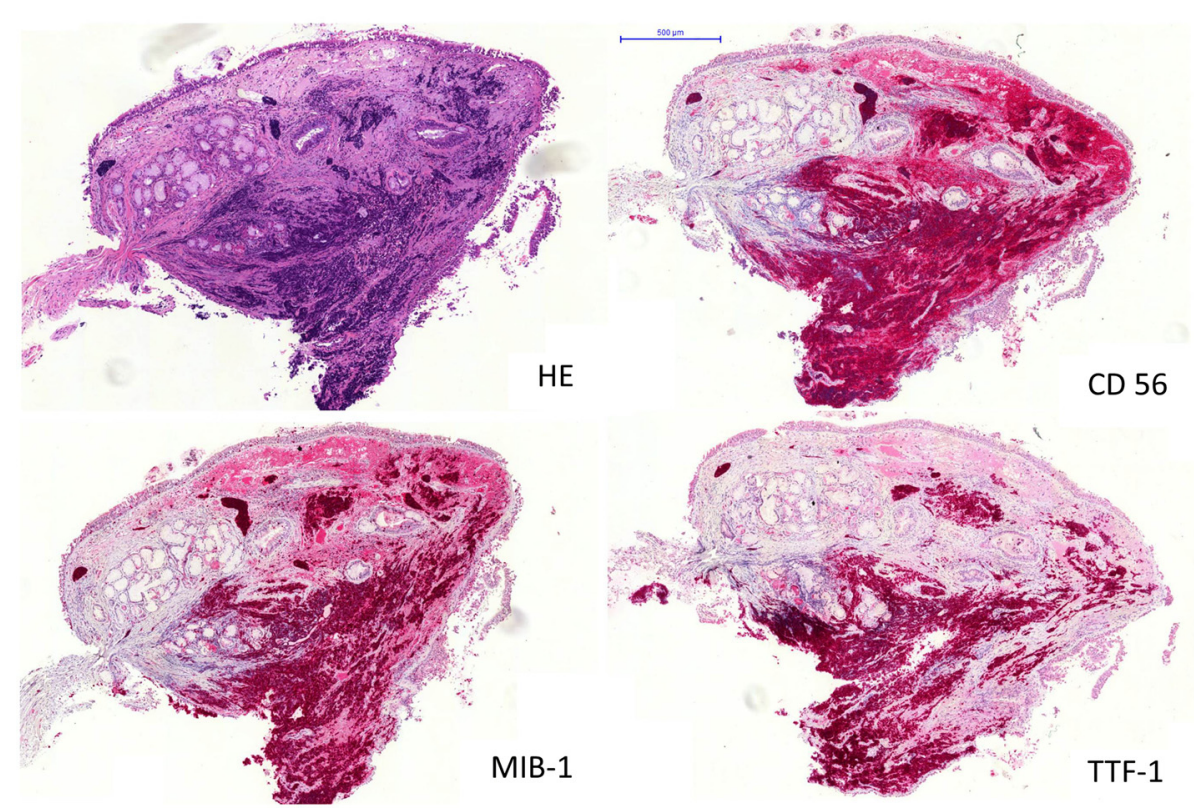

Figure 2 Example of TTF-1 positive small cell lung cancer (SCLC) staining. The tumor was identified as TTF-1 positive when more than 5\% of cells stained for TTF-1. Left upper panel: Hematoxylin and eosin stain (HE). Right upper panel: Neural cell adhesion molecule (CD56). Left lower panel: Ki-67 Proliferation marker (Clone MIB-1). Right lower panel: Thyroid transcription factor-1 (TTF-1).

Palliative irradiation was applied to patients for the local control of tumor deposits, i.e. of bone or brain metastases. Patients diagnosed in stage IV received preferably standard palliative chemotherapy as described above. All other lines of chemotherapy were noted until the patient died or cases were censored for this analysis. The number of lines is reported per patient. According to the treating physician best supportive care was applied in patient with low functional status and/or denied informed consent for therapy.

\section{Response to therapy}

Patients on treatment were followed-up on a regular basis, performing a chest CT and a CT of the upper abdomen (including the adrenal glands) every second cycle. The final staging for the response evaluation to first line chemotherapy was performed 3 weeks $+/-7$ days after completion of first line treatment. The response was defined according to RECIST 1.0 [18]: Complete Response (CR) meaning complete disappearance of all target lesions; Partial Response (PR) meaning at least 30\% reduction of the sum of all target lesions longest diameters; Progressive Disease (PD) meaning at least 20\% increase of the sum of all target lesions longest diameters, taking as reference the smallest measurements recorded since the treatment started and Stable Disease (SD) being defined as neither sufficient shrinkage to qualify for PR nor sufficient decrease to qualify for PD.

The disease control rate (DCR) was defined as all patients showing at least a stable disease after first line treatment whereas the overall response rate comprised only patients with a complete or partial response.

\section{Statistical analysis}

All frequencies are reported as number and percentage. Continuous variables are reported as mean \pm standard deviation. Frequencies were analyzed with Chi-square statistics with Fisher's exact test were needed. The disease control rate (DCR) was analyzed according to the effects of stage (III vs. IV) and TTF-1-status with a Chi-square test with layer analysis (Mantel-Haenszel statistics). Continuous survival was analyzed with Kaplan-Meier statistics and strata were compared with log-rank analyses. Survival time is reported as median days and the 95\% confidence interval. All analyses were carried out with the Statistical package for Social Sciences (SPSS Version 19.0) on a Microsoft Windows ${ }^{\oplus}$ operating system.

\section{Results}

Mean age of all patients was $64.1 \pm 10.2$ years $(n=294)$. A total of $119 / 294$ patients (40.5\%) had stage III (IIIA, $\mathrm{n}=32$, $10.9 \%$; IIIB, $\mathrm{n}=87,29.6 \%$ ) and $175 / 294$ patients (59.5\%) had stage IV SCLC according to the UICC-6 staging system.

Of the patients with stage III disease ( $n=119 / 294,40.5 \%$ ), $70 / 119(58.8 \%)$ received combined radio- and chemotherapy (simultaneous $\mathrm{n}=50,42.0 \%$; sequential, $\mathrm{n}=20,16.8 \%$; all platinum-based) as first line therapy, whereas $6 / 119$ patients (5.0\%) had chemotherapy with palliative radiotherapy (i.e. incomplete dose) only. 30/119 patients (25.2\%) received 
only chemotherapy and 8/119 (6.7\%) only radiotherapy (definitive $\mathrm{n}=3,2.5 \%$; palliative $\mathrm{n}=5,4.2 \%$ ). Best supportive care was applied in $4 / 119$ cases (3.4\%) and 1/119 therapies could not be retrieved. A total of 30/119 patients (25.2\%) had prophylactic brain irradiation followed by of systemic treatment.

In the population of the patients with stage IV ( $\mathrm{n}=175$ / 294, 59.5\%) small cell lung cancer, 160/175 (91.4\%) received palliative chemotherapy (platinum-based, $n=154 / 160$, 96\%) for systemic treatment. Out of these, 52/160 patients $(32.5 \%)$ additionally received palliative radiotherapy due to brain metastases $(n=24)$, bone metastases $(n=12)$ or for local control of thoracic tumor sites $(n=21)$. After palliative chemotherapy, $9 / 175$ patients $(5.1 \%)$ had prophylactic brain irradiation. $7 / 175$ patients $(4.0 \%)$ received only palliative radiotherapy. Best supportive care was administered in $4 / 175$ patients $(2.3 \%)$. No information on therapeutic regime was available for $2 / 175$ cases (1.2\%).

TTF-1 immunhistochemistry was available for 221/294 patients $(75.2 \%)$ of the entire population. Of these, 38/221 (17.2\%) had TTF-1 negative small cell lung cancer, whereas 183/221 (82.8\%) tumors showed reactivity in TTF-1 immunostaining. The distribution of gender was equal in both groups. Comparing the percentage of TTF-1 negative tumors according to stage, no significant difference was observed [Table 1].

Of the patients included into the per protocol analyses $181 / 221$ (81.9\%) died during the observation period. The median overall survival of all patients with known TTF1-status was 341 days (95\% CI 282-400 days). Patients with stage IV disease showed a significant shorter overall survival (290 [95\% CI 249-331] days) as compared to those with stage IIIA (488 [95\% CI 352-624] days) and IIIB disease (451 [95\% CI 354-548] days; $\mathrm{p}<0.001 \log$ rank analysis for all strata).

Stratifying for the TTF-1-status, median overall survival of all patients with TTF-1 positive tumors was 374 (95\% CI 306-442) days. Patients with TTF-1 negative SCLC had a median overall survival of 290 (95\% CI 191-389) days, which was not significantly different from those with
TTF-1 positive disease $(\mathrm{p}=0.254)$. When analyzing the overall survival according to TTF-1 for the different stage groups, no significant difference was observed for stage IV $(\mathrm{p}=0.237)$ as well as for IIIA $(\mathrm{p}=0.852)$ and IIIB patients $(\mathrm{p}=0.506)$ [Table 2 and Figure 3].

For the analysis of response to treatment according to TTF-1-status of the tumor, patients were excluded when no therapy or best supportive care $(n=18)$ was applied or response to treatment was not available $(n=25)$. Therefore, 178/221 (77.1\%) of all patients with known TTF-1-expression were eligible. Of these, $28 / 178$ patients (15.7\%) had TTF-1 negative SCLC.

Analyzing univariately the disease control rate (DCR), we observed a significantly $(p=0.013)$ improved response to treatment in the group of patients with TTF-1-expression (DCR 135/150 (90\%), CR, $\mathrm{n}=8$; PR, $\mathrm{n}=105 ; \mathrm{SD}, \mathrm{n}=22$; PD, $\mathrm{n}=15)$ as compared to those without TTF-1expression (DCR 20/28 (71.4\%); CR, $\mathrm{n}=4$; PR, $\mathrm{n}=16$; $\mathrm{SD}$, $\mathrm{n}=0 ; \mathrm{PD}, \mathrm{n}=8)$. Regarding the overall response rates (ORR) in the entire population of stage III and IV patients, the number of patients showing at least a partial remission was only slightly different between both groups (TTF-1-pos. 75.3\% vs. TTF-1-neg. 71.4\%) failing to reach the level of significance $(\mathrm{p}=0.642)$ [Table 3].

When the DCR was analyzed separately for nonmetastatic (stage III) and metastatic patients (stage IV), it was significantly higher in patients with TTF-1positive SCLC in stage IV (79/92, 85.9\%) compared to patients in the similar stage but with TTF-1-negative carcinoma $(10 / 18,55.6 \% ; p=0.006)$. This analysis did not show any difference in stage III patients because the DCR was very high for both groups of patients (DCR TTF-1pos. 56/58 96\% versus TTF-1-neg. 10/10 100\%; $\mathrm{p}=0.726$ ).

\section{Discussion}

TTF-1 is an immunhistochemical marker, which can help to differentiate between primary lung carcinoma and nonpulmonary cancer. In NSCLC, TTF-1-expression occurs mainly in adenocarcinoma and plays a crucial role in differentiating primary lung adenocarcinoma from pulmonary

Table 1 Patient characteristics

\begin{tabular}{|c|c|c|c|c|}
\hline & all & TTF1 + & TTF1 - & p-value \\
\hline Entire population & 221 & $183 / 221(82.8 \%)$ & $38 / 221(17.2 \%)$ & \\
\hline Age $($ mean $\pm S D)$ & $64.7 \pm 10.2$ & $64.0 \pm 10.2$ & $68.1 \pm 9.6$ & $0.023^{*}$ \\
\hline Female n (\%) & $76 / 221(34.4)$ & 63/183 (34.4\%) & 13/38 (34.2\%) & $1.000^{* *}$ \\
\hline Male n (\%) & $145 / 221(66.6)$ & 120/183 (65.6\%) & $25 / 38(65.8 \%)$ & \\
\hline Stage IIIA n (\%) & 21/221 (9.5) & 16/183 (8.7\%) & $5 / 38(13.2 \%)$ & \\
\hline Stage IIIB n (\%) & $64 / 221(29.0)$ & $56 / 183(30.6 \%)$ & $8 / 38(21.0 \%)$ & \\
\hline Stage IV n (\%) & $136 / 221(61.5)$ & $111 / 183(60.7 \%)$ & 25/38 (65.8\%) & $0.412^{* *}$ \\
\hline
\end{tabular}

Overview of the proportion of patients with TFF-positive and TTF1-negative SCLC in the entire patient population and in the different stage groups.

*Student's T-Test.

${ }^{* *}$ Chi-Square Test. 
Table 2 Overall survival (OS) in days according to TTF1 in the entire population and in the subgroups of patients with stage IIIA, IIIB and IV disease

\begin{tabular}{llll}
\hline & TTF1 + & TTF1 - & p-value* \\
\hline Entire population & $374(306-441)$ & $290(191-389)$ & 0.254 \\
Stage IIIA & $533(361-704)$ & $488(172-803)$ & 0.852 \\
Stage IIIB & $512(413-610)$ & $282(87-477)$ & 0.506 \\
Stage IV & $302(253-351)$ & $227(58-396)$ & 0.273 \\
\hline
\end{tabular}

*log-rank analyses.

metastatic disease [20]. In SCLC, the expression of TTF-1 was found to be very frequent in various studies. However, a review of the literature reveals that SCLC show a lack of TTF-1 expression in about $10-15 \%$ of all cases $([6,7,21-25])$. This is in line with the results of our study, where $17 \%$ of SCLC did not show TTF-1-expression. The frequency was neither associated with disease stage (III or IV), age nor gender.

Various studies investigated the immunhistochemical expression pattern of non-pulmonary small cell carcinoma, such as prostate or gastrointestinal SCC, revealing discrepant results. While Ordanez et al. found TTF-1expression in only 4 of 54 cases, another study by Yao et al. on 18 patients with small cell prostate carcinoma showed TTF-1-expression in $83 \%$ of cases $[10,11]$. This indicates that TTF-1 is not an exclusively pulmonological marker but rather points towards a neuroendocrine origin of the neoplastic cell $[1,12]$. This is strengthened by the fact that non-pulmonary SCC as well as SCLC express other neuroendocrine markers such as chromogranin A, neuro-specific enolase (NSE), CD57 or CD56 [26-31]. Therefore, the value of TTF-1 for origindiagnosis of small cell carcinoma seems to be dispensable, especially as the therapeutic approaches in cases of SCC do usually not differ according to the origin if the primary tumor site.

Another aspect of TTF-1-expression is a potentially possible predictive value on overall survival of lung cancer patients. For NSCLC, there seems to be evidence that patients with TTF-1 positive tumors show improved overall survival compared to those with TTF-1 negative tumors $[9,13-16,23]$. In our survival analyses including 221 SCLC patients, no significant difference could be shown for the comparison between patients with different TTF-1-expression. After stratifying for stage of disease, the results persisted in the subgroups of patients with stage III as well as stage IV disease.
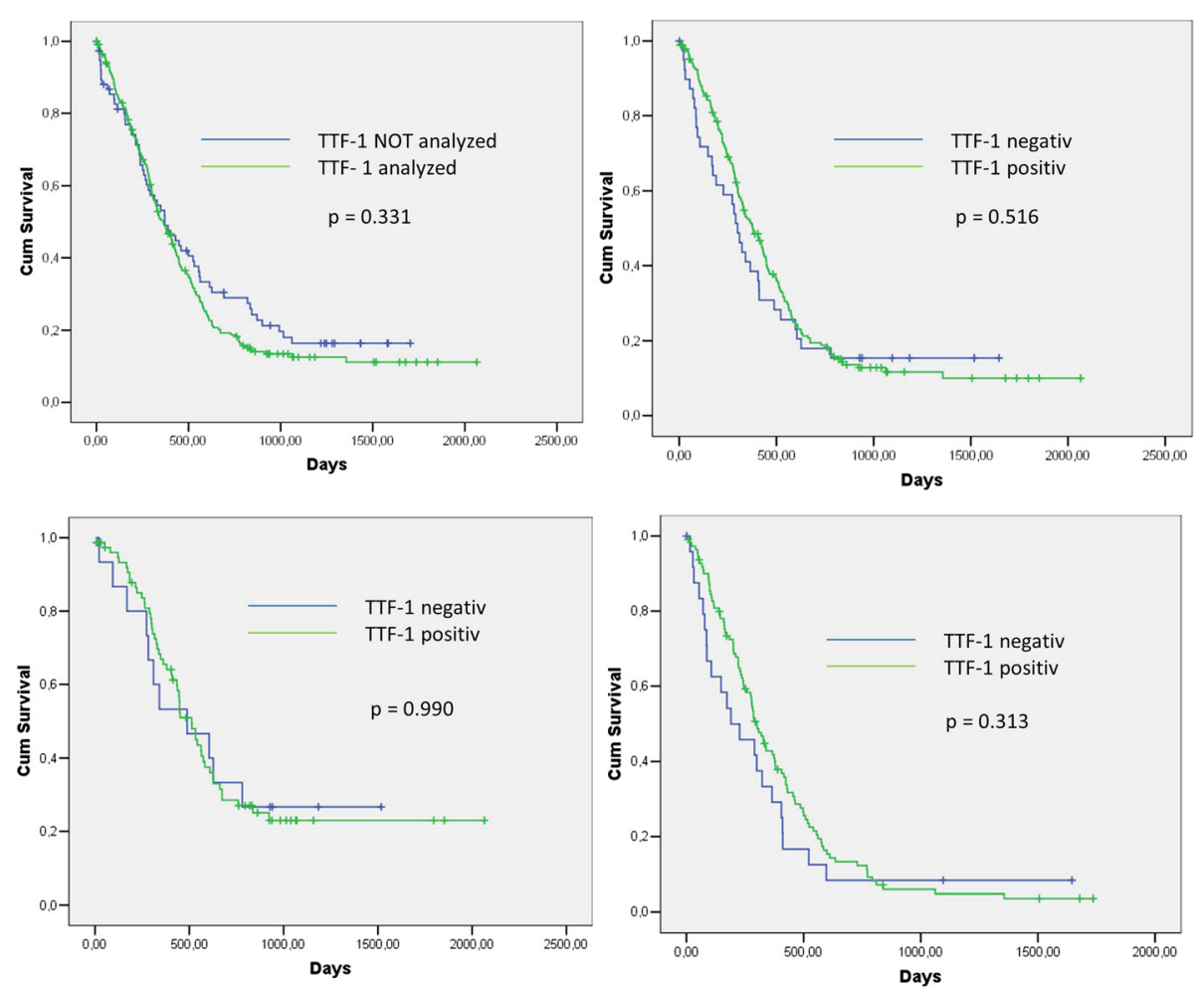

Figure 3 Survival Analysis and sub-analysis for disease stage according to TTF-1 (Kaplan-Meier plots with log-rank analysis; PP = per protocol, ITT = intention to treat). Left upper panel: ITT ( $n=294)$ vs. PP population $(n=221 ; p=0.331)$. Right upper panel: PP population: TTF-1 positive ( $n=183)$ vs. TTF-1 negative patients $(n=38 ; p=0.516)$. Left lower panel: PP sub-population with non-metastatic disease $(n=85)$ : TTF-1 positive $(n=72)$ vs. TTF-1 negative patients $(n=13 ; p=0.990)$. Right lower panel: PP sub-population with metastatic disease $(n=136)$ : TTF-1 positive $(n=111)$ vs. TTF-1 negative patients $(n=25 ; p=0.313)$. 
Table 3 Response to treatment according to TTF1-expression $(n=178)$

\begin{tabular}{llll}
\hline & TTF1+150 (84.3\%) & TTF1 $\mathbf{n = 2 8 ~ ( 1 5 . 7 \% )}$ & p-value \\
\hline CR & $8(5.3 \%)$ & $4(14.3 \%)$ & \\
PR & $105(70.0 \%)$ & $16(57.1 \%)$ & \\
SD & $22(14.7 \%)$ & 0 & \\
PD & $15(10.0 \%)$ & $8(28.6 \%)$ & $\mathbf{0 . 0 0 3}$ \\
RR & $75.3 \%$ & $71.4 \%$ & 0.642 \\
DCR (overall) & $135(90.0 \%)$ & $20(71.4 \%)$ & $\mathbf{0 . 0 1 3}$ \\
DCR stage III & $56 / 58(96.6 \%)$ & $10 / 10(100 \%)$ & 0.726 \\
DCR stage IV & $79 / 92(85.9 \%)$ & $10 / 18(55.6 \%)$ & $\mathbf{0 . 0 0 6}$ \\
\hline
\end{tabular}

Overall response rate (RR) is defined as the proportion of patients having at least a partial response according to RECIST1.0. Disease control rate (DCR) is defined as all patients with at least a stable disease according to RECIST1.0. $(\mathrm{CR}=$ complete response; $\mathrm{PR}=$ partial response; $\mathrm{SD}=$ stable disease; $\mathrm{PD}=$ progressive disease).

Regarding the response to therapy according to RECIST, the response rates of first line chemotherapy in the population of SCLC patients are known to be high in contrast to NSCLC. However, a number of approximately $10-20 \%$ of all SCLC does not respond to first line chemotherapy. The reason for that is mainly unknown. As the chemotherapy is known to cause significant side effects decreasing the quality of life of patients, it is of essential need to identify markers that may predict response to chemotherapeutic treatment. To our knowledge, there is currently no other study addressed to the possible implication of TTF-1-expression on response to chemotherapy in SCLC. In our analysis, a significantly higher disease control rate (DCR) in the group of TTF-1-positive SCLC could be shown, assuming a higher chemosensitivity of these tumors. Stratified by stage, this effect was highly significant in stage IV but could not be reproduced in stage III. We see this as a result of the high radiation rate in the stage III subgroup (overall 70.5\%), masking the presumed lower chemosensitivity of TTF-1-negative tumors.

Although this was a strong effect in metastatic disease, this difference did not result in a relevant overall survival benefit. Furthermore, response rates are still high in the subgroup of TTF-1-negative patients (>55\%), which does not allow a reliable identification of patients not benefiting from platinum based chemotherapy with TTF- 1 only. If TTF- 1 could be of predictive value used side-by-side with other biomarkers (i.e. MIB-1) should be investigated in further studies.

\section{Conclusions}

In summary, the diagnostic value of routine immunhistochemistry of TTF-1 in case of suspected SCLC appears to be low. Concerning the diagnostic aspect, the morphology of tumor cells and other immunhistochemical markers, such as chromogranin A, neuro-specific enolase (NSE), CD57 or CD56, are sufficient to enable the diagnosis of small cell cancer with neuroendocrine differentiation. As TTF-1 can be missing in case of SCLC and as well be present in case of non-pulmonary SCC, it seems to be of limited value for the definition of the primary tumor.

Concerning the prognostic aspect, our data indicate no prognostic implication of TTF-1-expression in SCLC patients. However, we observed a strong association between the absence of TTF-1-expression and the risk of first line failure in patients receiving platinum based chemotherapy only.

\section{Competing interests}

The authors declare that they have no competing interests.

\section{Authors' contributions}

DM and JK contributed to the design of the study, participated in the coordination of the study and the analysis of the data and helped to draft the manuscript, TB contributed to the design of the study and participated in the analysis of the data, CB, TW and CC participated in the coordination of the study and the analysis of the data, SG and TM performed the immunostaining of the tumors and contributed on the design of the study, TTB contributed to the design of the study, participated in the analysis of the data, performed the statistical analysis and helped to draft the manuscript. All authors read and approved the final manuscript.

\section{Acknowledgements}

The authors want to thank the Oskar-Helene-Stiftung for supporting the study. Furthermore, we thank Carmen Sommerfeld, Sabine Widdrat, Patricia Pflugmacher and Regina Peters for their substantial support of the organization and coordination of the study.

\section{Author details}

'Department of Pneumology, Lungenklinik Heckeshorn, HELIOS Klinik Emil von Behring, Walterhöferstr. 11, 14165 Berlin, Germany. ${ }^{2}$ Institute of Pathology, HELIOS Klinikum Emil von Behring, Berlin, Germany.

Received: 24 October 2014 Accepted: 11 March 2015

Published online: 02 April 2015

\section{References}

1. Bingle CD. Thyroid transcription factor-1. Int J Biochem Cell Biol. 1997;29(12):1471-3.

2. Lau SK, Luthringer DJ, Eisen RN. Thyroid transcription factor-1: a review. Appl Immunohistochem Mol Morphol. 2002;10(2):97-102.

3. Maeda Y, Dave V, Whitsett JA. Transcriptional control of lung morphogenesis. Physiol Rev. 2007;87(1):219-44.

4. Maeshima AM, Omatsu M, Tsuta K, Asamura H, Matsuno Y. Immunohistochemical expression of TTF-1 in various cytological subtypes of primary lung adenocarcinoma, with special reference to intratumoral heterogeneity. Pathol Int. 2008;58(1):31-7.

5. Lazzaro D, Price M, de Felice M, Di Lauro R. The transcription factor TTF-1 is expressed at the onset of thyroid and lung morphogenesis and in restricted regions of the foetal brain. Development. 1991;113(4):1093-104.

6. Zamecnik J, Kodet R. Value of thyroid transcription factor-1 and surfactant apoprotein $\mathrm{A}$ in the differential diagnosis of pulmonary carcinomas: a study of 109 cases. Virchows Arch. 2002;440(4):353-61.

7. Di Loreto C, Di Lauro V, Puglisi F, Damante G, Fabbro D, Beltrami CA. Immunocytochemical expression of tissue specific transcription factor-1 in lung carcinoma. J Clin Pathol. 1997;50(1):30-2

8. Yatabe Y, Mitsudomi T, Takahashi T. TTF-1 expression in pulmonary adenocarcinomas. Am J Surg Pathol. 2002;26(6):767-73.

9. Haque AK, Syed S, Lele SM, Freeman DH, Adegboyega PA. Immunohistochemical study of thyroid transcription factor-1 and HER2/neu in non-small cell lung cancer: strong thyroid transcription factor-1 expression predicts better survival. Appl Immunohistochem Mol Morphol. 2002;10(2):103-9. 
10. Ordonez NG. Value of thyroid transcription factor-1 immunostaining in distinguishing small cell lung carcinomas from other small cell carcinomas. Am J Surg Pathol. 2000;24(9):1217-23.

11. Yao JL, Madeb R, Bourne P, Lei J, Yang X, Tickoo S, et al. Small cell carcinoma of the prostate: an immunohistochemical study. Am J Surg Pathol. 2006;30(6):705-12.

12. Kitamura H, Yazawa T, Sato H, Okudela K, Shimoyamada H. Small cell lung cancer: significance of RB alterations and TTF-1 expression in its carcinogenesis, phenotype, and biology. Endocr Pathol. 2009;20(2):101-7.

13. Berghmans T, Paesmans M, Mascaux C, Martin B, Meert AP, Haller A, et al. Thyroid transcription factor 1-a new prognostic factor in lung cancer: a meta-analysis. Ann Oncol. 2006;17(11):1673-6.

14. Saad RS, Liu YL, Han H, Landreneau RJ, Silverman JF. Prognostic significance of thyroid transcription factor-1 expression in both early-stage conventional adenocarcinoma and bronchioloalveolar carcinoma of the lung. Hum Pathol. 2004;35(1):3-7.

15. Tan YK, Wee TC, Koh WP, Wang YT, Eng P, Tan WC, et al. Survival among Chinese women with lung cancer in Singapore: a comparison by stage, histology and smoking status. Lung Cancer. 2003:40(3):237-46.

16. Barlesi F, Pinot D, Legoffic A, Doddoli C, Chetaille B, Torre JP, et al. Positive thyroid transcription factor 1 staining strongly correlates with survival of patients with adenocarcinoma of the lung. Br J Cancer. 2005;93(4):450-2.

17. L.H. Sobin, M.K. Gospodarowicz, Ch. Wittekind (eds.): TNM Classification of Malignant Tumours, 7th Edition, Copyright 2009 by John Wiley \& Sons, New York.

18. Miller $A B$, Hoogstraten $B$, Staquet $M$, Winkler $A$. Reporting results of cancer treatment. Cancer. 1981;47(1):207-14.

19. Wu M, Wang B, Gil J, Sabo E, Miller L, Gan L, et al. p63 and TTF-1 immunostaining. A useful marker panel for distinguishing small cell carcinoma of lung from poorly differentiated squamous cell carcinoma of lung. Am J Clin Pathol. 2003;119(5):696-702.

20. Jagirdar J. Application of immunohistochemistry to the diagnosis of primary and metastatic carcinoma to the lung. Arch Pathol Lab Med. 2008;132(3):384-96.

21. Kargi A, Gurel D, Tuna B. The diagnostic value of TTF-1, CK 5/6, and p63 immunostaining in classification of lung carcinomas. Appl Immunohistochem Mol Morphol. 2007;15(4):415-20.

22. Fabbro D, Di LC, Stamerra O, Beltrami CA, Lonigro R, Damante G. TTF-1 gene expression in human lung tumours. Eur J Cancer. 1996;32A(3):512-7.

23. Myong NH. Thyroid transcription factor-1 (TTF-1) expression in human lung carcinomas: its prognostic implication and relationship with wxpressions of p53 and Ki-67 proteins. J Korean Med Sci. 2003;18(4):494-500.

24. Folpe AL, Gown AM, Lamps LW, Garcia R, Dail DH, Zarbo RJ, et al. Thyroid transcription factor-1: immunohistochemical evaluation in pulmonary neuroendocrine tumors. Mod Pathol. 1999;12(1):5-8.

25. Hiroshima K, lyoda A, Shida T, Shibuya K, lizasa T, Kishi H, et al. Distinction of pulmonary large cell neuroendocrine carcinoma from small cell lung carcinoma: a morphological, immunohistochemical, and molecular analysis. Mod Pathol. 2006;19(10):1358-68.

26. Wilson BS, Lloyd RV. Detection of chromogranin in neuroendocrine cells with a monoclonal antibody. Am J Pathol. 1984;115(3):458-68.

27. Gould VE, Lee I, Warren WH. Immunohistochemical evaluation of neuroendocrine cells and neoplasms of the lung. Pathol Res Pract. 1988;183(2):200-13.

28. Said JW, Vimadalal S, Nash G, Shintaku IP, Heusser RC, Sassoon AF, et al. Immunoreactive neuron-specific enolase, bombesin, and chromogranin as markers for neuroendocrine lung tumors. Hum Pathol. 1985;16(3):236-40.

29. Lantuejoul S, Moro D, Michalides RJ, Brambilla C, Brambilla E. Neural cell adhesion molecules (NCAM) and NCAM-PSA expression in neuroendocrine lung tumors. Am J Surg Pathol. 1998;22(10):1267-76.

30. Kibbelaar RE, Moolenaar KE, Michalides RJ, Van Bodegom PC, Vanderschueren RG, Wagenaar SS, et al. Neural cell adhesion molecule expression, neuroendocrine differentiation and prognosis in lung carcinoma. Eur J Cancer. 1991;27(4):431-5.

31. Kontogianni K, Nicholson AG, Butcher D, Sheppard MN. CD56: a useful tool for the diagnosis of small cell lung carcinomas on biopsies with extensive crush artefact. J Clin Pathol. 2005;58(9):978-80.

\section{Submit your next manuscript to BioMed Central and take full advantage of:}

- Convenient online submission

- Thorough peer review

- No space constraints or color figure charges

- Immediate publication on acceptance

- Inclusion in PubMed, CAS, Scopus and Google Scholar

- Research which is freely available for redistribution

Submit your manuscript at www.biomedcentral.com/submit 